\title{
El rendimiento académico de los estudiantes de las licenciaturas de médico cirujano y fisioterapia determinado por análisis predictivo
}

\author{
María Esther Urrutia Aguilar', Efrén Raúl Ponce Rosas², Silvia Ortiz León ${ }^{3}$, Laura Peñaloza Ochoa ${ }^{4}$ \\ y Rosalinda Guevara Guzmán ${ }^{5}$ \\ ${ }^{1}$ Facultad de Medicina, Universidad Nacional Autónoma de México; ${ }^{2}$ Subdivisión de Medicina Familiar; ${ }^{3}$ Departamento de Psiquiatría y Salud Mental; \\ ${ }^{4}$ Licenciatura en Fisioterapia; ${ }^{5}$ Departamento de Fisiología. Facultad de Medicina, Universidad Nacional Autónoma de México, Ciudad de México, México
}

\section{Resumen}

Objetivo: Identificar y comparar los factores predictores asociados al desempeño académico de los estudiantes de medicina que cursan la asignatura de "Biología celular e histología humana" y de los estudiantes de fisioterapia que cursan la asignatura de «Biología molecular, celular y tisular». Método: Se realizó seguimiento académico en el ciclo escolar, se aplicaron instrumentos de conocimientos previos, vocacionales, psicológicos y modos de afrontamiento al inicio del ciclo escolar; los dos últimos fueron aplicados dos veces más. Los datos fueron analizados considerando estadística descriptiva, comparativa, de correlaciones y de predicción. La participación de estudiantes fue voluntaria y se vigiló la confidencialidad de los datos.

PALABRAS CLAVE: Predicción. Aspectos emocionales. Modos de afrontamiento. Conocimientos previos. Biología celular.

\begin{abstract}
Objective: To identify and compare the predictive agents associated with medical students' academic performance that are undertaking cellular biology and human histology, as well as those physiotherapists that take molecular, cellular and tissue biology. Methods: An academic follow up was carried out during school. Tools on previous knowledge, vocation, psychological and confrontational means were applied at the beginning of the school year; and the last two were applied two more times afterwards. Data were analyzed considering descriptive, comparative, correlational and predictive statistics. The students' participation was voluntary and data confidentiality was looked after.
\end{abstract}

KEY WORDS: Prediction. Emotional aspects. Confrontational means. Previous knowledge. Cellular biology.

\section{Correspondencia:}

María Esther Urrutia-Aguilar

Edificio B-1er piso, Unidad de Posgrados

Ciudad Universitaria

Fecha de recepción: 07-08-2017

C.P: 04510, Ciudad de México

E-mail: mariau@unam.mx
Fecha de aceptación: 07-09-2017

DOI://dx.doi.org/10.24875/GMM.M17000012
Gac Med Mex. 2017;153;Sup 2:S119-S126

Contents available at PubMed www.anmm.org.mx 


\section{Introducción}

El Plan de Estudios 2010 de la carrera de médico cirujano que se imparte en la Facultad de Medicina de la Universidad Nacional Autónoma de México (UNAM) se caracteriza por ser un currículo mixto por asignaturas con un enfoque por competencias ${ }^{1}$, y se implementó en el año 2011. Su objetivo es formar médicos capaces y competentes para ejercer la medicina general de calidad en ambientes complejos y cambiantes.

En la misma Facultad de Medicina de la UNAM, el Consejo Universitario aprobó el 3 de febrero de 2014 la creación de la licenciatura en fisioterapia, que también se caracteriza por un currículo mixto; la primera generación ingresó en el ciclo escolar 2012-2013. El objetivo de esta carrera es formar fisioterapeutas poseedores de conocimientos científicos y actitudes de servicio, excelencia y profesionalismo que contribuyan a la restauración, preservación y potencialización del movimiento corporal humano.

Posterior a la implementación del Plan de Estudios 2010 (de médico cirujano) se ha observado un alto índice de alumnos que no acreditan las asignaturas del primer año, dando como consecuencia un considerable porcentaje de alumnos recursadores ${ }^{2}$, lo que ha motivado a varios académicos a desarrollar investigación educativa para dar cuenta de este problema. Así, se ha demostrado que los antecedentes académicos desempeñan un papel muy importante para acreditar las diferentes asignaturas ${ }^{3}$.

Otros factores que afectan el rendimiento académico son los señalados por algunos autores, como el estrés, la ansiedad y la depresión experimentados por los estudiantes durante el ciclo escolar. Estos factores originan un círculo vicioso en el estudiante, ya que son causa y consecuencia de problemas como el bajo desempeño académico ${ }^{4-12}$.

Anterior a este estudio, en la Facultad de Medicina de la UNAM se encontró que las variables que predicen el bajo desempeño son las relacionadas con las calificaciones obtenidas en el primer examen departamental y en conocimientos previos; después de éstas siguen las variables psicológicas ${ }^{12}$.

Dado lo anterior, es importante continuar con investigaciones educativas agregando otras variables, como los estilos con que los estudiantes confrontan los eventos negativos y fuentes de estrés a través de un proceso en el cual primero evalúan la situación de la amenaza, después evalúan las respuestas potenciales que pueden realizar y, por último, enfrentan o ejecutan las respuestas de afrontamiento. Dentro de este proceso hay dos formas de afrontamiento: uno enfocado al problema y otro a la emoción. Asimismo, los estilos de afrontamiento dependen de factores personales, determinando que los individuos que tienen una evaluación positiva de una situación estresante es más probable que posean el control de su medio ambiente ${ }^{13,14}$. De forma similar, Bandura ${ }^{15}$ ha descrito que las expectativas de eficacia son las que determinan en las personas la persistencia y el esfuerzo del afrontamiento; la aplicación de su teoría en el ámbito educativo se refiere a que los estudiantes con altas expectativas de autoeficacia gozan de motivación académica, obtienen mejores resultados, son más capaces de autorregular eficazmente su aprendizaje y muestran mayor motivación intrínseca en el mismo ${ }^{16,17}$.

También se ha encontrado relación entre la forma de afrontamiento y el rendimiento académico: las formas positivas se relacionan con un mejor rendimiento ${ }^{18}$.

Para realizar predicciones se necesita considerar diversos factores que afectan a cada individuo. El objetivo de este trabajo fue identificar y comparar los factores predictores asociados al desempeño académico de los estudiantes de medicina que cursan la asignatura «Biología celular e histología humana» y de los estudiantes de fisioterapia que cursan la asignatura «Biología molecular, celular y tisular».

\section{Método}

Estudio observacional, descriptivo y longitudinal. La población de estudio fueron los estudiantes de la generación 2015 de las carreras de médico cirujano y de fisioterapia.

La aplicación de los instrumentos de conocimientos previos, aspectos psicológicos, de modos de afrontamiento y de factores vocacionales se realizó durante la semana de inducción a la facultad (agosto de 2014), y la participación fue totalmente voluntaria. Además, se aplicaron los instrumentos psicológicos y de modos de afrontamiento en noviembre de 2014 y marzo de 2015. La interpretación de los resultados de los instrumentos de aspectos psicosociales y de afrontamiento fue realizada por psiquiatras y psicólogas.

El cálculo de la muestra se obtuvo con la siguiente ecuación:

$$
n=\frac{k^{2 *} p^{*} q^{*} N}{\left(e^{2 *}(N-1)\right)+k^{2 *} p^{*} q}
$$

con un nivel de confianza del $95 \%$. 


\section{Criterios de inclusión}

Estudiantes de la generación 2015 de las licenciaturas de médico cirujano y fisioterapia que contestaron todos los instrumentos (conocimientos previos, psicosociales, modos de afrontamiento y factores vocacionales) y que contaran con las calificaciones parciales de "Biología celular e histología humana" para los estudiantes de medicina y de «Biología molecular, celular y tisular» para los estudiantes de fisioterapia.

\section{Instrumentos}

\section{1) Factores académicos}

Los exámenes diagnósticos de conocimientos previos son elaborados por la Dirección General de Evaluación Educativa. Son pruebas integradas con preguntas de opción múltiple con cinco opciones de respuesta, de las cuales solo una es correcta. Se aplican dos exámenes: español e inglés con 60 preguntas cada uno y conocimientos generales con 120 preguntas que exploran las áreas de física, matemáticas, química, biología, historia y geografía.

Para el estudio del nivel del desempeño académico se tomaron en cuenta las calificaciones obtenidas en los exámenes departamentales de biología celular por los estudiantes de primer año, durante el ciclo escolar 2014-2015. Cabe mencionar que la evaluación de las asignaturas para los estudiantes de médico cirujano comprende la calificación de los exámenes departamentales, que son de opción múltiple y se califican en un rango de cero a diez puntos; su aplicación es de manera simultánea a todos los alumnos y el valor de este examen es del $50 \%$; el otro $50 \%$ es la calificación que el profesor asigna antes de cada examen departamental de acuerdo con sus propios criterios.

Para el caso de los estudiantes de fisioterapia, la calificación se otorga con un $50 \%$ juicio del profesor, un $30 \%$ teórico y un $20 \%$ práctica clínica.

2) Factores psicológicos

Para evaluar los aspectos psicológicos se solicitó a los estudiantes la firma del consentimiento informado. Se utilizaron dos instrumentos: A) Inventario de Beck para Depresión ${ }^{19,20}$ y B) Lista de síntomas SCL-

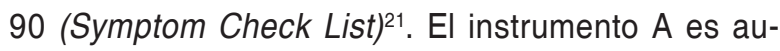
toaplicable y consta de 21 reactivos para evaluar síntomas depresivos en adolescentes y adultos; cada reactivo consiste en una serie de cuatro afirmaciones, de las cuales una tiene que ser seleccionada con relación a la forma en la que se ha sentido el paciente en la última semana. La puntuación total varía de 0 a 63 , pero solo se consideró una puntuación mayor o igual a 13 como punto de corte para identificar los casos con probable depresión. El instrumento B es una herramienta de tamizaje para identificar los síntomas de diversas psicopatologías y está compuesto por 90 reactivos con respuesta tipo Likert con cinco niveles de puntuación (0 a 4). En la validación del instrumento con población mexicana se encontró una adecuada consistencia interna, con alfa de Cronbach de todas las subescalas superior a 0.713. Para este trabajo, en el análisis se consideró solo la subescala de depresión con un punto de corte mayor o igual a 1.514 .

Las pruebas se aplicaron en tres momentos diferentes antes del periodo de los exámenes departamentales: el primero al ingreso en el mes de agosto, el segundo en el mes de noviembre y el tercero en el mes de marzo.

3) Modos de afrontamiento

Se utilizó el cuestionario de Modos de afrontamiento ${ }^{22}$ de la versión en español validada y estandarizada en jóvenes de 15 a 24 años, con 66 reactivos clasificados en seis factores:

- Flexibilidad de afrontamiento $(\alpha=0.90)$.

- Afrontamiento dirigido al problema $(\alpha=0.63)$.

- Pensamiento mágico $(\alpha=0.73)$.

- Distanciamiento $(\alpha=0.59)$.

- Búsqueda de apoyo social $(\alpha=0.84)$.

- Revaloración positiva $(\alpha=0.57)$.

La confiabilidad reportada para la escala total tuvo un alfa de Cronbach de 0.90. La calificación es por subescalas, en donde a cada una se le otorga un porcentaje ponderado respecto al uso del modo de afrontamiento.

Cabe destacar que tanto el instrumento de factores psicosociales como este fueron interpretados por psicólogas y psiquiatras del Departamento de Salud Mental de la misma facultad.

4) Factores vocacionales

El instrumento fue elaborado por la Dirección General de Orientación y Servicios educativos de la UNAM. Se evalúan puntos como el razonamiento abstracto, las aptitudes (mecánica y de discriminación de figuras), el reconocimiento de palabras, los intereses y algunos aspectos de personalidad, pero sobre todo la vocación con la que ingresan los alumnos a la Facultad de Medicina.

Se realizó estadística descriptiva e inferencial con el uso del programa SPSS v. 21.0. 
La predicción se llevó a cabo por análisis discriminante, que es un método multivariado para caracterizar la relación entre un grupo de variables predictoras y una variable de agrupación con un número relativamente pequeño de categorías, y crea una combinación lineal de las variables predictoras para calcular un puntaje discriminante para cada función.

La variable dependiente se clasificó en cuatro grupos dependiendo del promedio general obtenido por los estudiantes de medicina en la asignatura «Biología celular e histología humana» y por los estudiantes de fisioterapia en «Biología molecular, celular y tisular» $(0=$ bajo rendimiento, alumnos por debajo de 6 ; $1=6$ a $7.5 ; 2=7.6$ a 8.5; y $3=$ mayor de 8.5).

Las variables independientes fueron todas las variables de los instrumentos de aspectos psicológicos, vocacionales, conocimientos previos y modos de afrontamiento.

\section{Resultados}

\section{Médico cirujano}

La población de estudio de los estudiantes de medicina fue de 1236 , de los cuales el $65 \%$ eran mujeres. Para la licenciatura en fisioterapia se constituyó de 109 estudiantes, el 80\% mujeres. La edad promedio para ambas fue de 18 años.

Con los criterios de inclusión arriba mencionados se obtuvo que de la población de médico cirujano (1236 alumnos) se tomó una muestra representativa de 332 estudiantes con un nivel de confianza del 95\%; el tamaño de muestra fue de 294 alumnos. La muestra de estudiantes de fisioterapia fue de 109, con un nivel de confianza del $92 \%$; la $n$ representativa fue de 60 alumnos.

\section{Conocimientos previos}

Los histogramas (Fig. 1) que se muestran son de las calificaciones globales obtenidas por los estudiantes. Se observa que la media para los de medicina fue de 53.53, mientras que para los de fisioterapia fue de 46.63 , sobre un total de 100 puntos.

\section{Aspectos psicosociales}

En la figura 2 se muestran los resultados de no casos y del nivel de sintomatología depresiva (bajo, alto y muy alto), así como los que no contestaron en los tres momentos de aplicación.
Observando los datos anteriores, en la última aplicación se denota el aumento de los alumnos catalogados en los niveles alto y muy alto, y disminuyen los considerados no casos; además, aumentó la población que no contestó.

\section{Análisis discriminante}

En la tabla 1 se muestran los autovalores que representan el grado de eficacia relativa de cada función discriminante.

En la licenciatura de médico cirujano, los ítems con mayor carga en la aplicación del momento 1 y 2 son los que integran los aspectos psicosociales, como son incapacidad por nervios, llorar últimamente, pensamientos suicidas y la percepción de sí mismo. También, en los resultados existen variables relacionadas con el instrumento de factores vocacionales, como fueron la autoeficacia y la habilidad en aptitud mecánica. Asimismo, del instrumento de modos de afrontamiento solo la variable de dirigido positivo tuvo en el modelo una carga importante.

En la licenciatura en fisioterapia, ningún alumno se clasificó en el grupo cero (bajo rendimiento). El sistema eliminó, por falta de importancia en el modelo, las variables de los instrumentos de modos de afrontamiento y de factores psicosociales, dejando únicamente variables del examen diagnóstico y evaluación de factores asociados a la elección de carrera.

Analizando las variables predictoras que más carga arrojaron en el modelo, encontramos puntaje obtenido en conocimientos previos en matemáticas, historia y español, además de las variables de aspectos vocacionales, como fueron deseabilidad social, altruismo, aptitud mecánica e interés en biología y en física.

Por lo anterior, las variables más importantes para la clasificación son las que hacen referencia a los conocimientos generales adquiridos en el bachillerato, y por lo tanto el examen diagnóstico será de vital importancia para determinar el nivel de desempeño del alumno.

Hay que considerar que las variables de aspectos psicosociales y las de modos de afrontamiento van ligadas a aspectos emocionales, y que en esta licenciatura fueron excluidas del modelo.

En la tabla 2 se presentan el nivel de incidencia en la predicción del desempeño académico por tipo de licenciatura.

Ambas carreras tienen como segundo factor de importancia las variables del instrumento evaluación de factores asociados a la elección de carrera. 

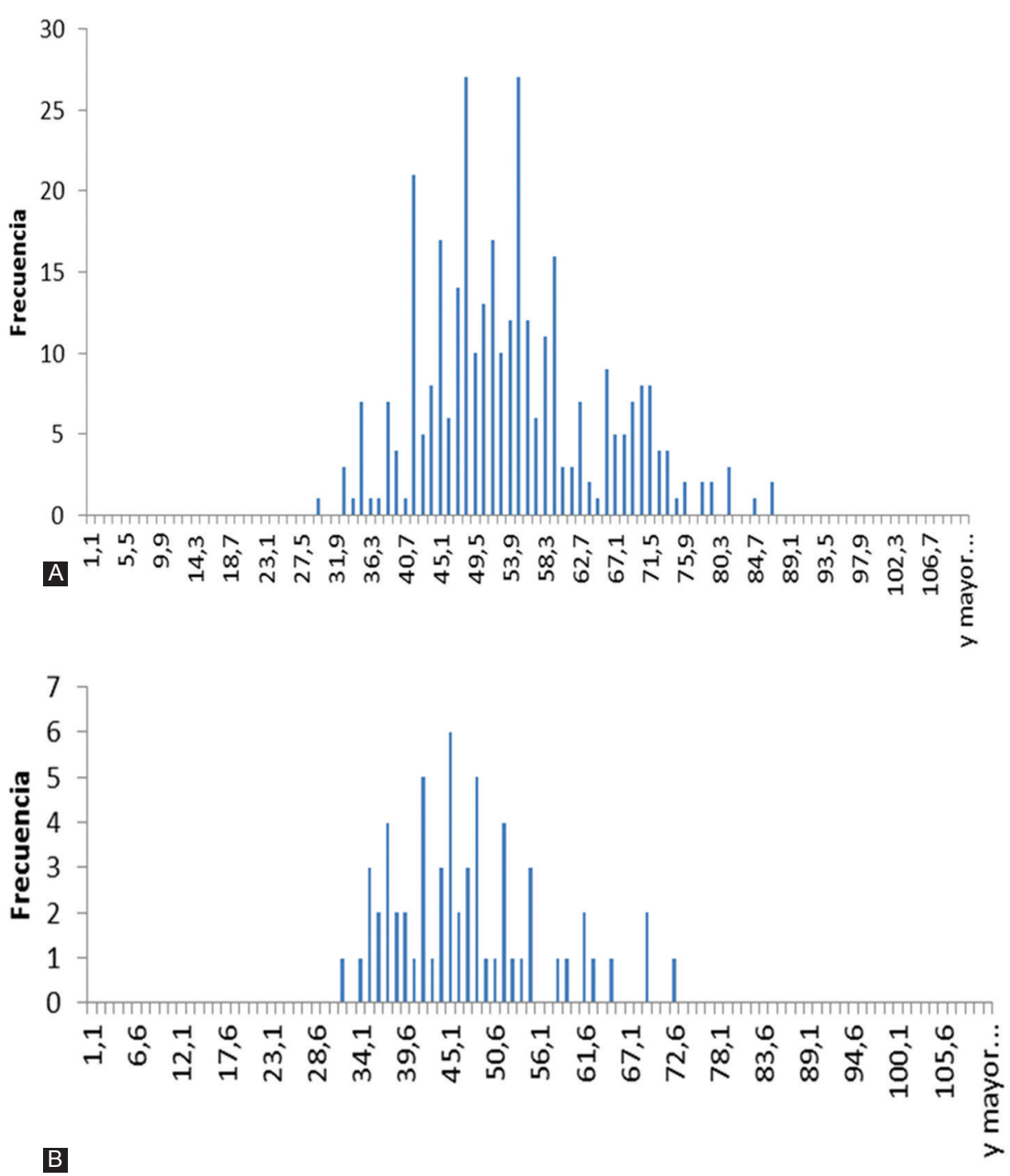

Figura 1. Histogramas de las calificaciones obtenidas en el examen general de conocimientos al ingreso de la licenciatura. A: médico cirujano. B: fisioterapia.

Con lo anterior se determina que, aunque existan diferencias en cuanto a las variables que determinan el desempeño académico en las licenciaturas de medicina y fisioterapia, existen ciertas variables que repercuten en el desempeño académico en ambas.

\section{Discusión}

Con los resultados obtenidos se contrastaron los modelos predictivos de desempeño académico de los estudiantes de las licenciaturas de médico cirujano y fisioterapia. Con ello se determinó que existen factores similares en ambas, como fueron algunas variables del instrumento de factores vocacionales.

El nivel de desempeño académico de los estudiantes de medicina estuvo determinado principalmente por variables contenidas en los instrumentos de factores psicosociales y factores asociados a la elección de carrera, y con menor carga las del examen diagnóstico y de modos de afrontamiento. En estudios anteriores con modelo multivariado en estudiantes de medicina que cursaban el primer año, las variables con más carga de predicción fueron los conocimientos previos, seguidas de los aspectos psicosociales ${ }^{12}$. En este estudio, los aspectos psicosociales fueron aplicados en tres momentos, a diferencia del anterior, en el que solo se aplicó dicho instrumento al iniciar el ciclo escolar, por lo que el seguimiento de los aspectos emocionales fue más determinante en este estudio. Estos resultados pueden deberse a la excesiva carga de trabajo que tienen los estudiantes, que va en aumento al transcurrir el año escolar, y a la necesidad de demostrar que tienen buen desempeño, 


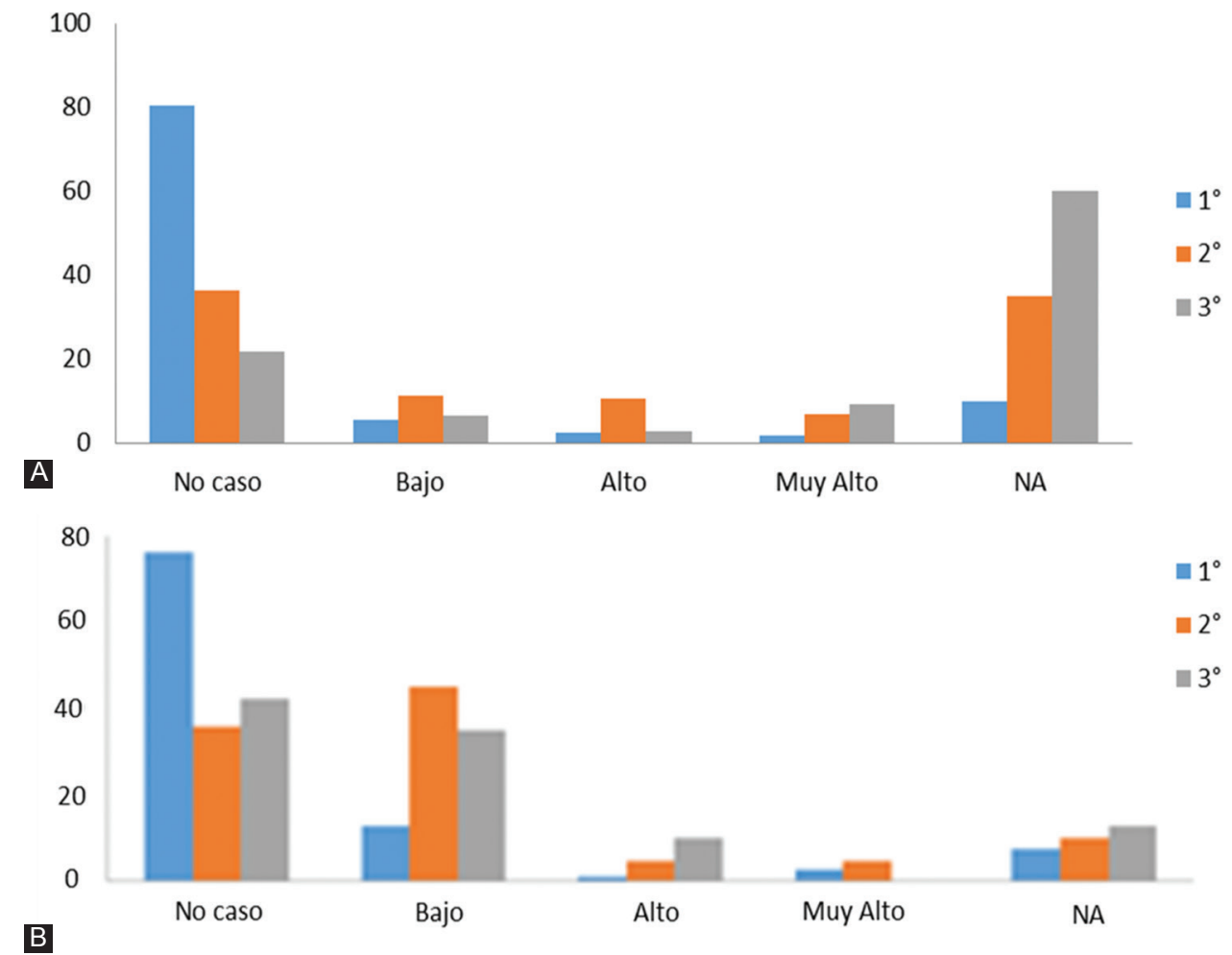

Figura 2. Porcentajes del nivel de depresión diagnosticado en los tres momentos de aplicación. A: médico cirujano. B: fisioterapia. NA: no contestaron el instrumento.

lo que puede repercutir en el deterioro de su salud mental. Por ello es de suma importancia considerar que encontrarse en una situación de desventaja crea en los estudiantes un sentimiento de fracaso que alimenta las fallas en su proceso de aprendizaje ${ }^{5}$.

Los datos más representativos indican que los síntomas de depresión en los alumnos de medicina son un factor asociado al bajo rendimiento académico. El nivel en el que los alumnos se sienten solos fue una variable determinante en el modelo predictivo, y se puede decir que el hecho de sentirse aislado repercute en el desempeño académico, ya que cuando los estudiantes utilizan estrategias de afrontamiento centradas en la evitación, como el distanciamiento social, tienen mayores consecuencias negativas, como depresión, ansiedad y percepción de una salud mental deficiente, y como consecuencia un mal rendimiento académico. En contraste, los que utilizan estrategias de solución de problemas, reinterpretación positiva, apoyo social y expresión de la emoción responden de una manera adaptativa, y por consiguiente reducen los efectos del estrés en su salud física y mental ${ }^{23}$.

En cuanto a los estudiantes que cursaban la licenciatura de fisioterapia, se observa que el modelo determinó que las variables del examen de factores psicosociales y las de modos de afrontamiento no fueron determinantes en las funciones, y por ello quedaron excluidas. Esto puede deberse a que los estudiantes que ingresan a esta licenciatura pasan varios filtros de ingreso, entre los que destacan una entrevista estructurada que es realizada por profesionales del área de la salud mental.

Las variables con más carga de predicción en los estudiantes de fisioterapia fueron las de conocimientos previos; en otros estudios se ha demostrado la importancia de ellos en el desempeño académico ${ }^{24}$.

Asimismo, en este grupo de estudiantes, las variables que también aportaron una carga importante en el modelo de predicción fueron las del instrumento de factores asociados a la elección de carrera. Esto puede deberse a que los estudiantes tienen como promedio de edad 18 años, lo que representa el final de la adolescencia, una etapa muy temprana para decidir su vocación.

En los estudiantes de medicina fue importante en el modelo discriminante la aptitud mecánica (habilidad para identificar los principios físicos que explican el movimiento de los cuerpos, el funcionamiento de los aparatos y el comportamiento de algunos fenómenos), y compartieron con los estudiantes de fisioterapia el 
Tabla 1. Resumen de las variables de la primera función discriminante por materia de médico cirujano

\begin{tabular}{|c|c|c|c|}
\hline \multicolumn{2}{|l|}{ Médico cirujano } & \multicolumn{2}{|l|}{ Fisioterapia } \\
\hline \multicolumn{2}{|l|}{ Biología celular e histología médica } & \multicolumn{2}{|l|}{ Biología molecular, celular y tisular } \\
\hline Variable & Función & Variable & Función \\
\hline Incapacidad a causa de nervios 2 & 8,956 & Matemáticas & 6.519 \\
\hline Sentirse mal contigo mismo 2 & 8,865 & Historia universal & 5.004 \\
\hline Mecánico & 8,174 & Deseabilidad social & -2.260 \\
\hline Pensamientos suicidas 1 & 7,812 & Español & -2.317 \\
\hline Sentimientos de culpa 1 & 7,713 & Organizacional/persuasivo & -1.190 \\
\hline Sentirme culpable por todo 2 & 7,551 & Aptitud mecánica & -1.185 \\
\hline Sentirme solo 1 & 7,463 & Mecánico & -1.132 \\
\hline Sentirse agobiado y en tensión 2 & 7,344 & Global inglés & -1.045 \\
\hline Dirig positivo & 7,011 & Historia de México & -1.005 \\
\hline Pensamientos suicidas 2 & 6,577 & Ciencias biológicas y de la salud & -0.936 \\
\hline Sentirse cansado 2 & $-6,863$ & Vocabulario & 1.071 \\
\hline Autoeficacia & $-7,575$ & Geografía & 1.120 \\
\hline Llorar últimamente 1 & $-7,701$ & Comprensión de lectura & 1.401 \\
\hline Dolores de cabeza 1 & $-8,281$ & Biología & 1.655 \\
\hline Descontento consigo mismo 2 & $-8,464$ & Química & 1.660 \\
\hline Interés por los demás 1 & $-9,073$ & Nivel de inglés & 1.697 \\
\hline Sentirse herido por los demás 2 & $-9,352$ & Ciencias físicas & 1.821 \\
\hline Clasificación Beck & $-10,123$ & Global & 2.168 \\
\hline Llorar últimamente 2 & $-13,121$ & Ciencias sociales & 3.265 \\
\hline Pensamientos suicidas 2 & $-16,098$ & Física & 6.397 \\
\hline
\end{tabular}

Nota: los números significan el tiempo de aplicación.

Tabla 2. Nivel de incidencia en la predicción según instrumento

Tipo de instrumento

Nivel de determinación en el modelo predictivo

Médico Fisioterapia

cirujano

\begin{tabular}{|c|c|c|}
\hline Examen diagnóstico & Bajo & Alto \\
\hline $\begin{array}{l}\text { Evaluación de factores asociados } \\
\text { a la elección de la carrera }\end{array}$ & Alto & Alto \\
\hline Examen de factores psicosociales & Alto & Nulo \\
\hline Modos de afrontamiento & Bajo & Nulo \\
\hline
\end{tabular}

área de interés en lo mecánico (preferencia por actividades relacionadas con conocer e investigar el funcionamiento de equipos e instalaciones, también gusto por armar y desarmar objetos). Lo anterior es importante debido a que para aprender biología celular es necesario tener capacidad para observar, describir la naturaleza con precisión, desarrollar y elaborar modelos, así como articular las habilidades cuantitativas para adquirir los conocimientos en biología celular ${ }^{25}$.

El análisis estadístico multivariado, específicamente el discriminante, es una herramienta que permite la identificación de los factores que predicen con una alta confiabilidad el desempeño académico en los estudiantes de las licenciaturas de médico cirujano y fisioterapia.

Finalmente, se recomienda continuar con el estudio de modelos multivariados de predicción, eliminando variables que puedan ser poco representativas para la discriminación de los grupos e incluyendo otras como pueden ser las variables socioeconómicas y de estrategias de estudio. 


\section{Bibliografía}

1. Sánchez-Mendiola M, Durante-Montiel I, Morales-López S, et al. Plan de Estudios 2010 de la Facultad de Medicina de la Universidad Nacional Autónoma de México. Gac Med Mex. 2011;147:152-8.

2. Informe Anual de Labores de la Facultad de Medicina 2012-2015. (Consultado el 6 de febrero de 2016.) Disponible en: http://www.facmed. unam.mx/_documentos/informe/2011/index.html

3. Guevara-Guzmán R, Galván M, Muñoz A. El desempeño académico de los estudiantes de la Facultad de Medicina en el primer año de la carrera. Gac Med Mex. 2007;143:27-32

4. Smith C, Peterson F, Degenhardt B, et al. Depression, anxiety, and perceived hassles among entering medical students. Psychol Health Med. 2007:12:31-9.

5. Fouilloux MC, Barragán PV, Ortiz LS, et al. Síntomas depresivos y rendimiento escolar en estudiantes de medicina. Salud Mental. 2013 36:59-65

6. Adams J. Straining to describe and tackle stress in medical students. Med Educ. 2004;38:463-4.

7. Dahlin M, Joneborg N, Runeson B. Stress and depression among medical students: a cross-sectional study. Med Educ. 2005;39:594-604.

8. Üner S, Öscebe H, Telatar G, et al. Assessment of mental health of university students with GHQ-12. Turkish Journal of Medical Sciences. 2008;38:437-46.

9. Omigbodun O, Odukogbe A, Akinyinka O, et al. Stressors and psychological symptoms in students of medicine and allied health professions in Nigeria. Soc Psychiatry Psychiatr Epidemiol. 2006;41:415-21.

10. Fouilloux $\mathrm{C}$, Petra I, Romero $\mathrm{M}$, et al. Fuentes de estrés en estudiantes de medicina. Revista Facultad de Medicina UNAM. 1994;37:132-6.

11. Urrutia-Aguilar ME, Varela RM, Vives VT, et al. Desafíos del universitario. México: Intersistemas; 2012

12. Urrutia-Aguilar ME, Ortiz LS, Foulloux MC, et al. El rendimiento académico en el primer año de la carrera de médico cirujano: modelo multivariado explicativo. Gac Med Mex. 2014;150:324-30.
13. Folkman S, Lazarus RS. An analysis of coping in a middleaged community sample. J Health Soc Behavior. 1980;21:219-39.

14. Folkman S, Lazarus RS. If it changes it must be a process: study of emotion and coping during three stages of a college examination. J Pers Soc Psychol. 1985;48:150-70.

15. Bandura A. Self-efficacy mechanism in human agency. Am Psychol. 1982;37:122-47.

16. Bandura A. Self-efficacy: toward a unifying theory of behavioral change. Psychol Rev. 1977;84:191-215

17. González MC, Tourón J. Autoconcepto y rendimiento académico. Sus implicaciones en la motivación y en la autorregulación del aprendizaje. Pamplona: EUNSA; 1982

18. Espinosa J, Contreras F, Esguerra G. Afrontamiento al estrés y modelo psicobiológico de la personalidad en estudiantes universitarios. Revista Diversitas-Perspectivas en Psicología. 2009:5:87-96.

19. Beck AT, Steer RA, Garbin MC. Psychometric properties of the Beck Depression Inventory. Twenty-five years of evaluation. Clin Psychol Rev. 1988;8:77-100.

20. Jurado S, Villegas ME, Méndez L, et al. La estandarización del Inventario de Depresión de Beck para los residentes de la ciudad de México. Salud Mental. 1998;21:26-31.

21. Cruz FCS, López BL, Blas GC, et al. Datos sobre la validez y confiabilidad de la Symptom CheckList 90 (SCL90) en una muestra de sujetos mexicanos. Salud Mental. 2005;28:72-81.

22. Sotelo CM, Maupone V. Traducción y estandarización del cuestionario Modos de afrontamiento al estrés de Lazarus y Folkman, para una población de adolescentes mexicanos. México, D.F.: Facultad de Psicología, Universidad Nacional Autónoma de México; 2000.

23. Rosenthal TL, Rosenthal RH, Edwards NB. Students' self-ratings of stress in medical school: a replication across 20 months. Behav Res Ther. 1990;28:171-3.

24. Amini M, Dehghani MR, Kojuri J, et al. A qualitative study of factors associated with medical students' academic success. J Soc Sci. 2008;4:347-51.

25. Aikens LM, Dolan LE. Teaching quantitative biology: goals, assessments, and resources. Mol Biol Cell. 2014;25:3478-81. 